\title{
Use of gene expression and whole-genome sequence information to improve the accuracy of genomic prediction for carcass traits in Hanwoo cattle
}

Sara de las Heras-Saldana ${ }^{1 \dagger}$, Bryan Irvine Lopez ${ }^{2 \dagger}$, Nasir Moghaddar ${ }^{1}$, Woncheoul Park ${ }^{2}$, Jong-eun Park ${ }^{2}$, Ki Y. Chung ${ }^{3}$, Dajeong Lim² ${ }^{2^{*}}$, Seung H. Lee ${ }^{4}$, Donghyun Shin ${ }^{5}$ and Julius H. J. van der Werf ${ }^{1^{*}}$ (D)

\begin{abstract}
Background: In this study, we assessed the accuracy of genomic prediction for carcass weight (CWT), marbling score (MS), eye muscle area (EMA) and back fat thickness (BFT) in Hanwoo cattle when using genomic best linear unbiased prediction (GBLUP), weighted GBLUP (wGBLUP), and a BayesR model. For these models, we investigated the potential gain from using pre-selected single nucleotide polymorphisms (SNPs) from a genome-wide association study (GWAS) on imputed sequence data and from gene expression information. We used data on 13,717 animals with carcass phenotypes and imputed sequence genotypes that were split in an independent GWAS discovery set of varying size and a remaining set for validation of prediction. Expression data were used from a Hanwoo gene expression experiment based on 45 animals.
\end{abstract}

Results: Using a larger number of animals in the reference set increased the accuracy of genomic prediction whereas a larger independent GWAS discovery dataset improved identification of predictive SNPs. Using pre-selected SNPS from GWAS in GBLUP improved accuracy of prediction by 0.02 for EMA and up to 0.05 for BFT, CWT, and MS, compared to a $50 \mathrm{k}$ standard SNP array that gave accuracies of $0.50,0.47,0.58$, and 0.47 , respectively. Accuracy of prediction of BFT and CWT increased when BayesR was applied with the $50 \mathrm{k}$ SNP array (0.02 and 0.03, respectively) and was further improved by combining the $50 \mathrm{k}$ array with the top-SNPs (0.06 and 0.04 , respectively). By contrast, using BayesR resulted in limited improvement for EMA and MS. WGBLUP did not improve accuracy but increased prediction bias. Based on the RNA-seq experiment, we identified informative expression quantitative trait loci, which, when used in GBLUP, improved the accuracy of prediction slightly, i.e. between 0.01 and 0.02 . SNPs that were located in genes, the expression of which was associated with differences in trait phenotype, did not contribute to a higher prediction accuracy.

\footnotetext{
*Correspondence: lim.dj@korea.kr; jvanderw@une.edu.au

tSara de las Heras-Saldana, Bryan I Lopez joint first authors

1 School of Environmental and Rural Science, University of New England,

Armidale NSW 2351, Australia

${ }^{2}$ Animal Genomics and Bioinformatics Division, National Institute

of Animal Science, Rural Development Administration, Wanju 55365,

Republic of Korea

Full list of author information is available at the end of the article
}

(c) The Author(s) 2020. This article is licensed under a Creative Commons Attribution 4.0 International License, which permits use, sharing, adaptation, distribution and reproduction in any medium or format, as long as you give appropriate credit to the original author(s) and the source, provide a link to the Creative Commons licence, and indicate if changes were made. The images or other third party material in this article are included in the article's Creative Commons licence, unless indicated otherwise in a credit line to the material. If material is not included in the article's Creative Commons licence and your intended use is not permitted by statutory regulation or exceeds the permitted use, you will need to obtain permission directly from the copyright holder. To view a copy of this licence, visit http://creativeco mmons.org/licenses/by/4.0/. The Creative Commons Public Domain Dedication waiver (http://creativecommons.org/publicdomain/ zero/1.0/) applies to the data made available in this article, unless otherwise stated in a credit line to the data. 
Conclusions: Our results show that, in Hanwoo beef cattle, when SNPs are pre-selected from GWAS on imputed sequence data, the accuracy of prediction improves only slightly whereas the contribution of SNPs that are selected based on gene expression is not significant. The benefit of statistical models to prioritize selected SNPs for estimating genomic breeding values is trait-specific and depends on the genetic architecture of each trait.

\section{Background}

Korean beef production is mainly based on the Hanwoo breed and production efficiency has improved significantly since the beginning of 1983 when performance and progeny testing was introduced in the Hanwoo breeding program [1]. In particular, marbling score has improved considerably due to combining improved feeding strategies (feeding more concentrates and for extended periods in the feedlot) and breeding strategies such as sire selection and artificial insemination. The high value of Hanwoo meat has motivated further genetic improvement of economically important carcass traits such as marbling score (MS), eye muscle area (EMA), back fat thickness (BFT), and carcass weight (CWT). In recent years, with the availability of new genomic and bioinformatics tools, approaches that use genotype information for the selection of Hanwoo cattle are being introduced because it can increase rates of genetic gain due to increased selection accuracy and decreased generation interval for the selection of breeding bulls [1]. Merban et al. [2] investigated the accuracy of genomic prediction for carcass traits in Hanwoo cattle by evaluating different models. They found that the accuracy of prediction for CWT increased when using BayesC compared with genomic best linear unbiased prediction (GBLUP) and Bayesian LASSO, but that there was no difference in accuracy between the three methods for BFT, EMA, and MS [2]. However, in this study sample size (1183 animals) and single nucleotide polymorphisms (SNP) density $(34,000$ SNPs) were considered to be limiting factors. The availability of sequence information and an increased number of genotyped animals provide scope for improving accuracy of prediction. Imputed full sequence information applied in standard GBLUP procedures does not necessarily improve the accuracy of prediction or explain more genetic variation of the traits compared with the use of $50 \mathrm{k}$ or high-density (HD) SNP arrays [3-5]. A more efficient use of sequence information can be achieved by selecting SNPs based on their effect on phenotypic differences or on knowledge of the biology of the traits, i.e. which genes are likely involved, and such information could be obtained from association studies or from other sources of information such as gene expression experiments.

In dairy cattle $[4,6,7]$ and sheep [8], pre-selection of SNPs from whole-genome sequence data based on either candidate genes or genome-wide association studies (GWAS) have resulted in small to moderate improvements in accuracy of prediction. GWAS in Hanwoo have provided information about the genetic architecture of carcass traits [9-14] and sensory traits [15]. In general, these studies suggest that carcass traits are polygenic since numerous quantitative trait loci (QTL) regions were detected, each with a small effect $[14,16]$. However, only a few QTL overlap between these studies in Hanwoo, which is likely due to the relatively small datasets used to identify QTL regions and the low to moderate marker density. QTL detection and identification of predictive SNPs can be improved with larger datasets and high-density genotype information, e.g. at the sequence level.

The use of more biological information (gene expression, methylation, protein, and metabolite data, among others) has been recommended to identify genomic features that are enriched for causal variants in complex traits. In dairy cattle, the addition of predictive SNPs for milk traits increased the accuracy by $16 \%$ in the Australian Red breed. In particular, prioritization of SNPs in coding and regulatory regions in a BayesRC model increased the accuracy of prediction for milk traits compared to using only high-density genotypes [17]. In another study, using information from the best-performing gene ontology term in a genomic feature BLUP (GFBLUP) model increased, on average, accuracy of prediction for milk traits (milk, fat, protein, and mastitis) by 0.02 points [18]. Xiang et al. [19] found that the identification of informative SNPs (high-ranking variants) based on a functional and evolutionary trait heritability (FAETH) score from multiple sources of information resulted in higher heritability and increased accuracy of prediction than the use of low ranking variants in Holstein and Danish Red cattle [19].

Information on genes that are involved in marbling phenotypes in Hanwoo cattle has been generated from a time-series gene expression experiment using quantitative PCR (qPCR) [20-23] and whole-transcriptome analysis and has led to the identification of eQTL and SNPs located in genes that are associated with marbling [24]. The aim of our study was to assess the prediction accuracy of GBLUP, wGBLUP, and Bayesian models for carcass traits (CWT, MS, EMA, and BFT) and the potential gain from using pre-selected SNPs from a GWAS on 
imputed sequence data and from gene expression information in Hanwoo cattle.

\section{Methods}

\section{Animals and phenotypes}

All the procedures described in this paper were in accordance with the protocols accepted by the Animal Care and Use Committee of the National Institute of Animal Science in the Republic of Korea (Approval No. 2018-293). The genotypes and phenotypes from 13,717 Hanwoo cattle were collected by the Animal Genomics and Bioinformatics Division of the National Institute of Animal Science (RDA) between 2000 and 2016 in commercial slaughterhouses in South Korea. Four carcass traits were analyzed; cold carcass weight (CWT, $\mathrm{kg}$ ) was recorded for each animal after $24 \mathrm{~h}$ of chilling whereas marbling score (MS), eye muscle area (EMA, $\mathrm{cm}^{2}$ ), and subcutaneous back fat thickness (BFT, $\mathrm{mm}$ ) were measured on the longissimus dorsi muscle between the 13th rib and the 1st lumbar vertebra. Marbling scores were assigned based on the Korean Beef Marbling visual standard category system (KAPE, 2012), which accounts for the percentage of intramuscular fat (IMF) observed from 1 (no IMF) to 9 (19\% or more IMF).

\section{RNA-seq analysis}

The transcriptomic analysis was performed on 45 Hanwoo steers, which were fed high (23 steers) and low (22 steers) energy diets. Longissimus dorsi muscle was sampled by biopsy between the 10th and 13th cervical vertebra at $7,8,12,18$, and 24 months of age, and collected after slaughter at 30 months of age. For the biopsies, each steer was restrained in a hydraulic squeezed chute, hair was removed from the biopsy site, and local anesthetic (lidocaine $\mathrm{HCl} ; 20 \mathrm{mg} / \mathrm{mL} ; 8 \mathrm{~mL}$ per biopsy site) was administered. The biopsy site was cleaned with $70 \%$ ethanol on sterile surgical gauze and a 1 to $3 \mathrm{~cm}$ skin incision was made with a sterile scalpel. Tissue ( 1 to $2 \mathrm{~g}$ ) was collected from the muscle using a sterile Bergstrom biopsy needle $(5.3 \mathrm{~mm}$ diameter) and was preserved in liquid nitrogen and stored at $-80^{\circ} \mathrm{C}$. After closing the incision site with veterinary tissue glue, the area was covered with a spray-on aluminum bandage. All steers were monitored for swelling 24 and $48 \mathrm{~h}$ after biopsy. Samples were obtained on alternate sides of the animal depending on the location of the previous sampling, and the third and fourth biopsies were sampled $5 \mathrm{~cm}$ away from the location of the previous biopsy.

RNA was extracted from the isolated tissues using the TRIzol reagent (Invitrogen, Life Technology, Carlsbad, USA) following the manufacturer's recommendations. The quality of the RNA was evaluated on a Bioanalyzer 2100 with RNA 6000 Nano Labchips
(Agilent Technologies Ireland, Dublin). The high-quality RNA samples with an average RNA integrity value higher than 7 were used to produce indexed shotgun paired-end (PE) libraries with on average 500 bp inserts generated using a TruSeq Nano DNA Library Prep Kit (Illumina, USA) following the standard Illumina sample-preparation protocol. The resulting libraries were sequenced on an Illumina HiSeq 2500 sequencer $(2 \times 101$ bp pairedend sequences).

The quality of the reads was assessed with the FastQC tool [25], low-quality bases (Phred $<33$ ) and adapters were removed using Trimmomatic v0.32 [26]. We mapped the resulting reads to the Bos taurus reference genome (version UMD 3.1) using the HISAT2 v2.1.0 [27] software and the $\mathrm{R}$ function featureCount from the package Rsubread v1.34.7 to obtain the gene counts [28].

\section{Genotypes and imputation}

The $50 \mathrm{k}$ SNP genotypes and sequences were obtained from an Illumina platform and SNP locations were derived using the Bos taurus reference genome version UMD 3.1. Quality control thresholds were set to filter out SNPs with a minor allele frequency lower than 0.01 , that deviated from Hardy-Weinberg disequilibrium $\left(p<10^{-6}\right)$ and to exclude individuals with more than $5 \%$ missing genotypes. A subset of 4566 animals (4452 of those with recorded phenotypes for carcass traits) with previously imputed whole-sequence genotypes, based on 203 fully sequenced Hanwoo animals, were used as a reference for imputing genotypes of the remaining 10,215 samples to high-density and whole-sequence genotype data. Genotypes were phased with the software Eagle v2.4.1 [29] and we used Minimac3 [30] to impute the $50 \mathrm{k}$ genotypes $(58,991$ SNPs) of 10,215 animals to high-density (HD; 543,263 SNPs) and finally up to whole-sequence $(10,723,697 \mathrm{SNPs})$. The final imputed sequence data (that included only SNPs with a Minimac3 $R^{2}>0.4$ ) consisted of 10,723,697 SNPs with an average imputation accuracy $\left(R^{2}\right)$ of 0.99 from Minimac3.

\section{Selection of the discovery datasets for GWAS}

In order to select the statistically most significant SNPs to improve accuracy of genomic prediction, an association study is required. For this purpose, a discovery set needs to be extracted from the data that is independent of the set used for training and validating accuracy of prediction [17]. A larger discovery set allows a more accurate association study but leaves a smaller cross-validation set to train and test predictions. We evaluated the impact of using different numbers of animals in the discovery set on the identification of SNPs associated with carcass traits and the accuracy of prediction in Hanwoo cattle. To achieve this, four discovery datasets $(1000,2000$, 
3000 , and 4000 animals) were extracted from the complete dataset of 13,717 animals. We selected animals for the discovery set such that the genetic diversity in each set was high by selecting the set (out of one hundred random samples) that had the smallest average co-ancestry according to the following criterion:

$$
\sum_{i=1}^{n} \frac{\mathbf{x}^{\prime} \mathbf{G} \mathbf{x}}{2}
$$

where $\mathbf{x}$ is an indicator vector of the selected animals ( 1 or 0 if selected, or not), and $\mathbf{G}$ is the genomic relationship matrix for all the animals. The genomic relationship matrix was calculated with $50 \mathrm{k}$ SNP genotypes from all the animals using the Plink v1.90b4 software [31].

\section{Selection of cross-validation datasets}

After selecting a discovery set, the remaining animals were used for a tenfold cross-validation $(\mathrm{CV})$ to evaluate the accuracy and bias of genomic prediction. Depending on the number of animals used in the discovery dataset, between 9717 and 12,717 animals were available for CV. The effect of forming the 10 subsets for $\mathrm{CV}$, randomly or by k-means clustering, was assessed by comparing the impact of these strategies on accuracy of prediction. Note that there were no sire-son relationships in the data, since all phenotypes were on commercially slaughter animals. However, sib relationships might exist, but no pedigree was available. Therefore, samples were grouped based on a k-mean strategy using the kmeans $\mathrm{R}$ function from the stats package, which basically uses as input the principal components matrix of the genomic relationships (from the $50 \mathrm{k} \mathrm{SNP} \mathrm{chip)} \mathrm{to} \mathrm{cluster} \mathrm{the} \mathrm{samples} \mathrm{with} \mathrm{a} \mathrm{smaller}$ within-cluster variation. The number of clusters was set to 100 , whereas the number of random starting partitions was 50 . Based on the assigned clusters, the samples were then split into 10 groups with a similar number of animals. It is expected that the $\mathrm{k}$-means clustering results in a lower degree of genetic relatedness between the validation and training sets, leading to a lower accuracy of genomic prediction but to a greater benefit from using selected SNPs in the prediction model.

To compare the different prediction methods with the selected SNPs, we decided to use a k-means strategy for selecting the validation and reference sets. This strategy decreased the relatedness between training and validation sets, which likely resulted in a greater benefit from using pre-selected SNPs from prediction [8] and therefore allowed a better comparison of methods. Moreover, in breeding programs, often the animals for which breeding values need to be predicted are relatively less related to the nucleus, and such a situation can be simulated with the k-means strategy.

\section{Statistical analysis}

All the traits were adjusted for fixed effects based on a univariate analysis in ASReml v4.1 [32]. The evaluated fixed effects were birth-year (15 levels: 2000 to 2016), birth-month (12 levels), age at slaughter (from 17 to 173 months), slaughter-year (9 levels: 2008 to 2018), slaughter-month (12 levels); slaughter-place (53 levels), herds of origin (324 levels), and sex (2 levels).

The fixed effects that were significant for all traits were herd of origin, birth-year, birth-month, slaughteryear, slaughter-month, slaughter-place, age, and sex. The interaction effect of herd $\times$ birth-year was fitted in a final model for CWT without fitting the main effects whereas a herd $\times$ birth-year $\times$ birth-month effect was fitted for MS and BFT. The adjusted phenotypes were obtained as the residuals from the fitted model for each carcass trait.

\section{Pre-selection of SNPs and GWAS}

A genome-wide association analysis (GWAS) was applied to each of the carcass traits based on each of the individual variants from the imputed sequence data, fitting a univariate linear mixed model using the GCTA v1.26.0 software [33]:

$$
\mathbf{y}=\mathbf{1} \mu+\mathbf{x}_{i} \alpha_{i}+\mathbf{Z a}+\mathbf{e},
$$

where $\mathbf{y}$ is the vector of $\mathrm{N}$ adjusted phenotypic values (for MS, BFT, EMA, or CWT), 1 is a vector with $\mathrm{N}$ ones, $\mu$ is the intercept, $\mathbf{x}_{i}$ is a vector of the genotypes at a single SNP $i, \alpha_{i}$ is the regression coefficient for the allele substitution effect of SNP $i, \mathbf{Z}$ is an incidence matrix for animals, $\mathbf{a}$ is vector of the random additive genetic effects of animals, and $\mathbf{e}$ is a vector of random residual effects; $\operatorname{var}(\mathbf{a})=\mathbf{G} \sigma_{\mathrm{a}}^{2}$ where $\mathbf{G}$ is the genomic relationship matrix based on the imputed sequence genotypes and $\operatorname{var}(\mathbf{e})=\mathbf{I} \sigma_{\mathrm{e}}^{2}$.

For each trait, significant SNPs were selected based on seven thresholds for the $-\log _{10}(p$ value) $(1.5,2,2.5$, $3,3.5,4$, or 5 , respectively). For each list of top SNPs, the software Plink was used to prune SNPs that were in high linkage disequilibrium (LD) with other SNPs in the region. SNPs with an $\mathrm{r}^{2}$ value higher or equal to 0.95 with the most significant SNP in a window of 5000 SNPs were removed, and the process was repeated after each window shift of 100 SNPs.

The proportion of genetic variance was calculated for significant SNPs as:

$$
\frac{2 p_{i}\left(1-p_{i}\right) \alpha_{i}^{2}}{\sigma_{a}^{2}} \times 100
$$

where $p_{i}$ is the allele frequency of the reference allele of SNP $i, \sigma_{a}^{2}$ is the additive genetic variance of the trait, and $\alpha$ is the estimated additive effect of SNP $i$. 


\section{Pre-selection of SNPs and gene expression}

Two strategies were followed to preselect SNPs from expression studies: (1) SNPs in genes the expression of which was associated with the traits, and (2) SNPs from expression QTL (eQTL). To evaluate the association of each gene expression with each of the traits (MS, CWT, EMA, and BFT) a linear regression of gene count on phenotype was used by fitting the feeding treatment and sires as fixed effect. We selected a significance threshold $p$-value $<0.0032\left(-\log _{10} p\right.$-value $\left.=2.5\right)$ to identify genes that were significantly associated (GSA) with a trait. For each GSA, relevant SNPs were identified in the imputed sequence data from the promoter region (300 bp before the transcription start site) to the end of the gene.

For the identification of eQTL, an association analysis of each SNP with each gene expression (13,572 genes) was performed with the $\mathrm{R}$ package MatrixEQTL v2.2 [34] using all imputed sequences SNPs of 45 animals and the gene expression at each of the five ages. The effect of the genotype on expression was assumed to be additive linear (modelLINEAR), by fitting the feeding treatments and sires as fixed effects in the model. The local (cis-) and distal (trans-) eQTL were identified using a significance threshold of $p$-value $<0.0032$.

The lists of eQTL were pruned in the same way as the GWAS SNPs by using Plink to remove the SNPs in high LD $\left(r^{2} \geq 0.95\right)$ with the most significant SNPs in a window of 5000 SNPs and repeating the process after shifting the window by 100 SNPs.

\section{Genomic prediction}

We used the MTG2 v9.09 software [35] to estimate variance components with restricted maximum likelihood (REML) and to calculate the genomic breeding values (GBV) using all the data that were not included in the GWAS discovery dataset. The accuracy of prediction was assessed for two GBLUP models:

$$
\begin{aligned}
& \mathbf{y}=\mathbf{1} \mu+\mathbf{Z g}_{1}+\mathbf{e} \\
& \mathbf{y}=\mathbf{1} \mu+\mathbf{Z g}_{1}+\mathbf{Z g}_{2}+\mathbf{e}
\end{aligned}
$$

where $\mathbf{y}$ is the vector of $\mathrm{N}$ adjusted carcass trait phenotypes (MS, CWT, EMA, and BFT), $\mathbf{1}$ is a vector with $\mathrm{N}$ ones, $\mu$ is intercept, $\mathbf{Z}$ is the design matrix to assign $\mathbf{y}$ to $\mathbf{g}$ (1 or 2), $\mathbf{g}_{1}$ and $\mathbf{g}_{2}$ are the additive genetic effects of individuals with $\operatorname{var}\left(\mathbf{g}_{i}\right)=\mathbf{G}_{i} \sigma_{a}^{2}$, and $\mathbf{e}$ is the vector of residual effects. Standard $50 \mathrm{k}$ SNP chip $(40,933$ SNPs) genotypes were used to calculate the $\mathbf{G}_{1}$ used for $\mathbf{g}_{1}$ (Model 3), while the pre-selected SNPs (top SNPs with $p<0.0032$ from GWAS or SNPs from GSA and eQTL) were used in a second $\mathbf{G}_{2}$ as a covariance matrix for $\mathbf{g}_{2}$ in Model 3, and in this case the overlapping top SNPs were removed from $\mathbf{G}_{1}$ and the resulting matrix is referred to as $\mathbf{G}_{\text {50adj }}$ The sum of $\mathbf{g}_{1}$ and $\mathbf{g}_{2}$ is the estimated breeding value (GBV) in Model 3.

A weighted-GBLUP (wGBLUP) procedure was implemented for the reference-validation dataset of 10,717 animals. This procedure is described in detail in [36]. Briefly, in the wGBLUP method, the $\mathbf{G}$ matrix is constructed by using the weight of each SNP and this weight is based on the estimated effect of the SNP on the trait, as derived from back-solving for SNP effects from the estimated genomic breeding values [36, 37], and recalculating these through five iterations. Following VanRaden [38], SNP weights were calculated as $\mathrm{d}_{\mathrm{i}(\mathrm{t}+1)}=1.25^{\frac{\left|\hat{\alpha}_{i}\right|}{\operatorname{sd}(\hat{\alpha})}-2}$, where $\alpha$ are estimated SNP effects in a previous iteration round.

A Bayesian mixture model was fitted by applying a Markov chain Monte Carlo (MCMC) method with 50,000 iterations (20,000 iterations were burn-in) to estimate parameters using the BayesR method as implemented by [39]. The model uses a mixture of four distributions for the effects of SNPs with variances in each distribution being 0, 0.0001, 0.001, and 0.01 of $\sigma_{g}^{2}$, respectively, $\sigma_{g}^{2}$ being estimated from the data. The BayesR model for prediction was:

$$
\hat{y}=\mu+\sum_{j \in \beta_{j}>0} w_{i j} \hat{\beta}_{J}
$$

where $\hat{\beta}_{j}$ is the estimated effect of SNP $j, w_{i j}=\frac{\left(x_{i j}-2 p_{j}\right)}{\sqrt{2 p_{j}\left(1-p_{j}\right)}}$ and $x_{i j}$ is the number of copies of the reference allele in the reference genome $(0,1,2)$ at SNP $j$ for individual $i$ and $p_{j}$ is the frequency of the reference allele.

Eight strategies were followed to compare the use of pre-selected SNPs in the estimation of breeding values for the carcass traits: (1) GBLUP-50 k: Model 2 with only the standard $50 \mathrm{k}$ array to calculate the genomic relationship matrix $\left(\mathbf{G}_{1}\right)$; (2) GBLUP-GWAS: using Model 3 with the top SNPs derived from GWAS to construct $\mathbf{G}_{2}$ along with the adjusted $50 \mathrm{k} \mathbf{G}\left(\mathbf{G}_{50 \text { adj }}\right)$ as $\mathbf{G}_{1}$; (3) GBLUPeQTL: using Model 3 with the selected eQTL to form the $\mathbf{G}_{2}$ together with the adjusted $50 \mathrm{k} \mathbf{G}\left(\mathbf{G}_{50 \text { adj }}\right)$ as $\mathbf{G}_{1}$; (4) GBLUP_GSA: using Model 3 with SNPs identified in GSA and differentially expressed (DE) genes to form the $\mathbf{G}_{2}$ in combination with the $50 \mathrm{k} \mathrm{G}\left(\mathbf{G}_{50 \mathrm{adj}}\right)$; (5) wGBLUP-50 k: fitting a weighted-GBLUP procedure applied to Model 2 using the standard $50 \mathrm{k} \mathrm{SNP}$ array to form $\mathbf{G}_{1}$; (6) wGBLUP-GWAS: using the standard $50 \mathrm{k}$ and top SNPs in one genomic relationship matrix as $\mathbf{G}_{1}$ in Model 2 and applying a wGBLUP procedure; (7) BayesR-50 k: performing a BayesR analysis with the standard $50 \mathrm{k}$ SNP array; and (8) BayesR-GWAS: using a BayesR model with genomic information that includes the standard $50 \mathrm{k}$ SNP array as well as the top SNPs. 


\section{Accuracy of prediction}

The accuracy of prediction was assessed as the Pearson's correlation coefficient ( $r$ ) between the adjusted phenotype of the animals in the validation dataset and their GBV divided by the square root of the heritability for each trait. The empirical standard error (SE) was calculated by dividing the standard deviation of the $10 \mathrm{calcu}-$ lated accuracies from the tenfold CV by the square root of 10 . The bias of the genomic prediction was calculated as the deviation from unity of the regression coefficient (b) of the phenotype on the GBV.

\section{Results}

The basic statistics and the estimated variance components and heritability for EMA, BFT, CWT, and MS are in Table 1 for Model 1 and Table 2 for Model 2. The heritabilities ranged from 0.24 to 0.27 . The sum of the two additive genetic components of the variance in Model 2 tended to be somewhat smaller than the additive genetic component in Model 1.

\section{Genomic prediction of carcass traits Comparison of $k$-means or random selection strategies for cross-validation}

The accuracy of genomic selection and the added value of using top SNPs likely depends on the genetic diversity of the population and the degree of relatedness between reference set and validation set. Two sampling strategies were compared for selecting the validation and reference sets: k-means clustering and random sampling. In total, 13,717 Hanwoo cattle were used for the comparison of accuracy of prediction for the carcass traits based on the standard $50 \mathrm{k}$ panel in a tenfold $\mathrm{CV}$.

Table 1 Descriptive statistics and variance components (standard error in brackets) estimated for the carcass traits in Hanwoo cattle $(n=13,717)$

\begin{tabular}{lllll}
\hline & BFT & EMA & CWT & MS \\
\hline$h^{2}$ & $0.24(0.01)$ & $0.24(0.01)$ & $0.25(0.01)$ & $0.27(0.01)$ \\
$\sigma_{a}^{2}$ & $4.8(0.33)$ & $27.6(1.80)$ & $571.4(36.44)$ & $0.66(0.04)$ \\
$\sigma_{p}^{2}$ & $20.1(0.28)$ & $113.5(1.56)$ & $2272.5(31.30)$ & $2.44(0.03)$ \\
$\sigma_{e}^{2}$ & $15.3(0.26)$ & $85.9(1.47)$ & $1701.1(29.18)$ & $1.77(0.03)$ \\
Min & 1 & 22 & 152 & 1 \\
Max & 57 & 156 & 692 & 9 \\
Mean & 13.42 & 92.61 & 425.50 & 5.68 \\
SD & 5.23 & 12.56 & 59.84 & 1.98 \\
\hline
\end{tabular}

$h^{2}$ : estimated heritability; $\sigma_{a}^{2}$ : additive genetic variance; $\sigma_{p}^{2}$ : phenotypic variance; $\sigma_{e}^{2}$ : residual variance; SD: standard deviation

Max maximum value, Min minimum value of adjusted phenotypes for BFT back fat thickness, EMA eye muscle area, CWT carcass weight and MS marbling score
Table 2 Variance components (standard error in brackets) for two G matrices estimated for the carcass traits in Hanwoo cattle $(n=10,717)$

\begin{tabular}{rlrl}
\hline & $\boldsymbol{h}^{\mathbf{2}}$ & $\boldsymbol{\sigma}_{\boldsymbol{a}}^{2}$ & \multicolumn{1}{c}{$\boldsymbol{\sigma}_{\boldsymbol{e}}^{\mathbf{2}}$} \\
\hline $\mathrm{BFT}$ & & & \\
$\mathbf{G}_{1}$ & $0.15(0.02)$ & $2.85(0.34)$ & $15.15(0.29)$ \\
$\mathbf{G}_{2}$ & $0.07(0.01)$ & $1.30(0.20)$ & \\
$\mathrm{EMA}$ & & & \\
$\mathbf{G}_{1}$ & $0.16(0.02)$ & $18.42(2.00)$ & \\
$\mathbf{G}_{2}$ & $0.05(0.01)$ & $5.73(1.21)$ & \\
$\mathrm{CWT}$ & & & \\
$\mathbf{G}_{1}$ & $0.12(0.02)$ & $251.95(34.72)$ & $1.83(1.66)$ \\
$\mathbf{G}_{2}$ & $0.08(0.01)$ & $177.46(25.15)$ & \\
$\mathrm{MS}$ & & & \\
$\mathbf{G}_{1}$ & $0.18(0.02)$ & $0.43(0.04)$ & \\
$\mathbf{G}_{2}$ & $0.06(0.01)$ & $0.14(0.03)$ & \\
\hline
\end{tabular}

$h^{2}$ : estimated heritability; $\sigma_{a}^{2}$ : additive genetic variance; $\sigma_{e}^{2}$ : residual variance; $\mathbf{G}_{1}$ : adjusted $\mathbf{G}$ matrix with $50 \mathrm{k}$ SNP array (top SNPs removed); $\mathbf{G}_{2}$ : $\mathbf{G}$ matrix with the top-SNPs from the 3000 discovery dataset

BFT back fat thickness, EMA eye muscle area, CWT carcass weight, MS marbling score

The accuracy of prediction of breeding values for carcass traits was, on average, 0.06 higher when using a random sampling strategy to perform the cross-validation compared to using a k-means clustering strategy (average accuracy is 0.58 vs 0.52 ; Fig. 1a). This is likely due to predicting animals in the validation set that are less related to the training set when selection is based on the k-means strategy. The difference between the sampling strategies was similar for all traits (ranging from 0.04 to 0.07 ). The bias was low (from -0.02 to 0.005 ) when the random strategy was used for the sampling, while bias was higher with the k-means strategy (ranging from 0.05 to 0.13 ) (Fig. 1b).

\section{Comparison of the accuracy of genomic predictions based on different dataset sizes}

The accuracy of prediction for each size of the referencevalidation dataset is in Table 3. For this comparison, we used GBLUP with one $\mathbf{G}$ matrix $\left(\mathbf{G}_{1}\right.$ in Model 2$)$ and the $50 \mathrm{k}$ SNP array. For all traits, with the larger referencevalidation (RV) dataset $(n=12,717)$ accuracies were higher (by 0.02-0.04) than with the smaller RV dataset $(\mathrm{n}=9717)$. These differences were not statistically significant but they were consistent across all traits. The reduction in the accuracy of prediction for the RV dataset with 10,717 animals was small and allowed us to use an independent discovery dataset of 3000 animals. Therefore, the RV dataset of 10,717 animals was used to compare different methods for the genomic prediction analysis. 

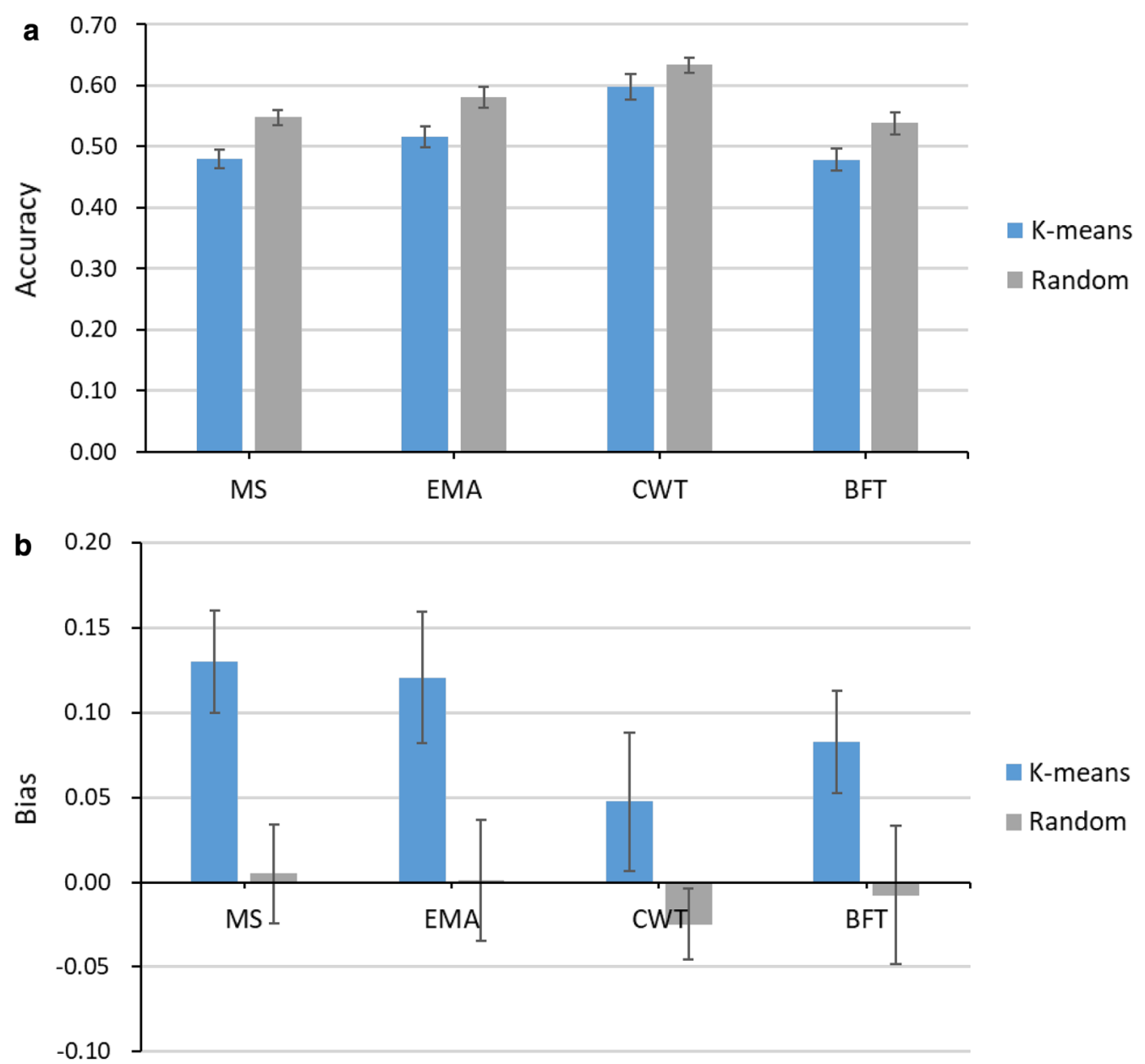

Fig. 1 Accuracy (a) and bias (b) of genomic prediction of breeding value for the carcass traits marbling score (MS), eye muscle area (EMA), carcass weight (CWT) and back fat thickness (BFT), using the standard $50 \mathrm{k}$ array for the $\mathrm{k}$-means and random selection cross-validation (CV). Vertical lines indicate the empirical standard error for each CV result

Table 3 Accuracies of prediction of breeding value (empirical CV standard error in brackets) for carcass traits in Hanwoo cattle using different sizes of referencevalidation (RV) datasets

\begin{tabular}{lllll}
\hline Trait & Dataset size & & & \\
\cline { 2 - 5 } & $\mathbf{R V}=\mathbf{1 2}, \mathbf{7 1 7}$ & $\mathbf{R V}=\mathbf{1 1 , 7 1 7}$ & $\mathbf{R V}=\mathbf{1 0 , 7 1 7}$ & $\mathbf{R V = 9 7 1 7}$ \\
\hline MS & $0.50(0.02)$ & $0.47(0.01)$ & $0.47(0.02)$ & $0.46(0.02)$ \\
EMA & $0.53(0.02)$ & $0.51(0.02)$ & $0.50(0.02)$ & $0.50(0.02)$ \\
CWT & $0.59(0.03)$ & $0.56(0.02)$ & $0.58(0.03)$ & $0.57(0.02)$ \\
BFT & $0.47(0.02)$ & $0.46(0.02)$ & $0.47(0.02)$ & $0.43(0.03)$ \\
\hline
\end{tabular}

BFT back fat thickness, EMA eye muscle area, CWT carcass weight, MS marbling score

\section{Pre-selection of SNPs based on GWAS}

To investigate what is the optimal number of preselected top SNPs for genomic prediction, we evaluated the increase in accuracy of prediction when a second $\mathbf{G}$ matrix $\left(\mathbf{G}_{2}\right)$ based on the selected SNP set was included in a GBLUP model for each trait (Fig. 2). We compared seven significant threshold values for SNP selection, with the $-\log _{10}(p$-value) equal to $5,4,3.5,3,2.5,2$ and 1.5 , and using the independent discovery set of 3000 animals. The number of significant SNPs increased rapidly with lower threshold values whereas accuracy tended to be highest when the largest SNP set was used. There was only a limited amount of overlap of the SNPs identified between the discovery datasets, and it was largest for CWT (see Additional file 1: Figure S1).

A threshold of $2.5\left(-\log _{10}(0.0032)\right)$ resulted in the nearly highest improvement in accuracy of prediction for carcass traits although a moderate number of top SNPs ( 8688) was used in $\mathbf{G}_{2}$ (Fig. 2). Therefore, this threshold was used to select the top SNPs from GWAS and to compare methods. Although an increase in accuracy 

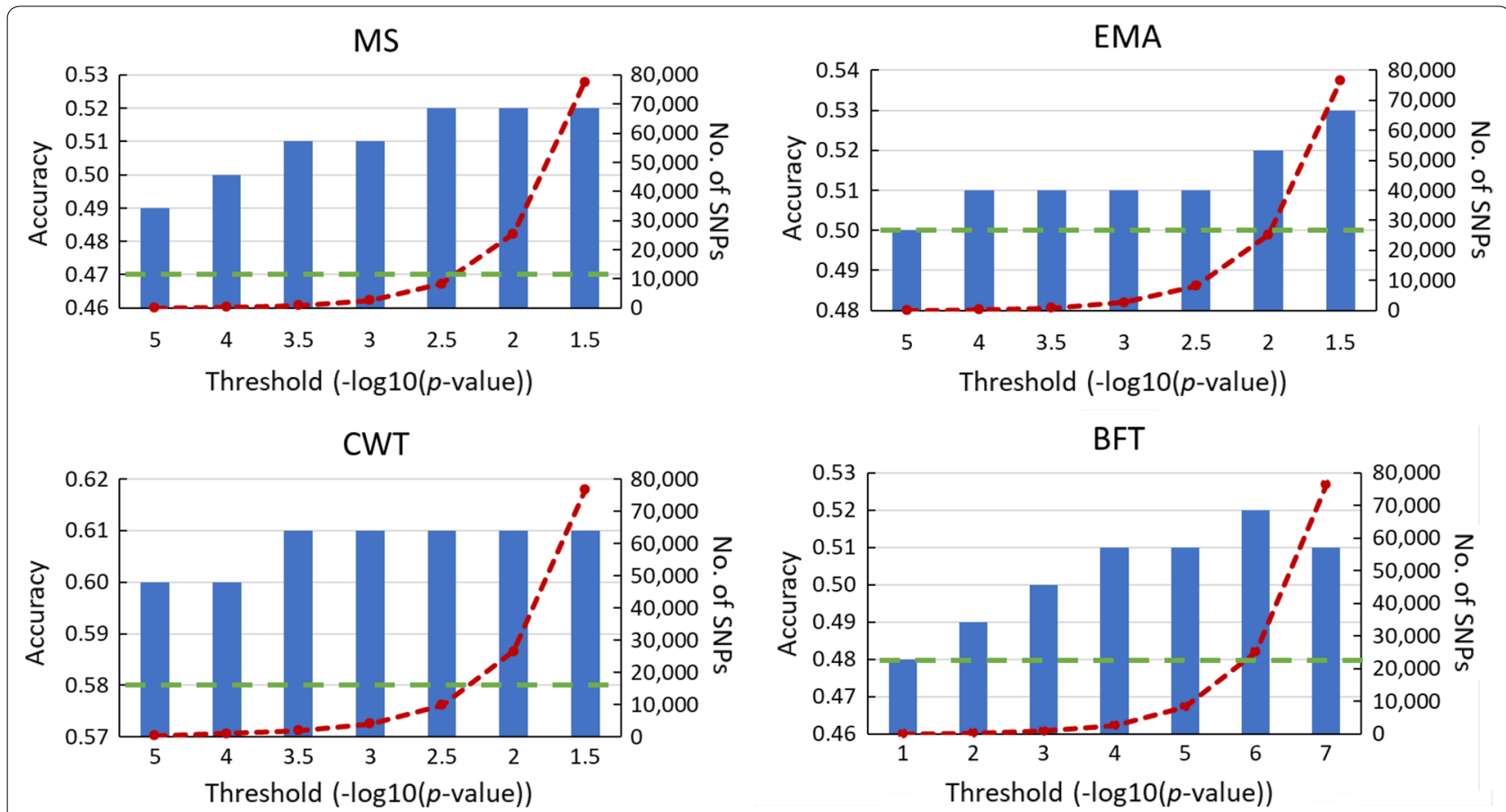

Fig. 2 Accuracy of genomic prediction of breeding value (bars) for carcass traits marbling score (MS), eye muscle area (EMA), carcass weight (CWT) and back fat thickness (BFT) by using a $50 \mathrm{k}$ standard SNP array and top SNPs from GWAS (3000 animals) added with various significance thresholds (red dashed line). The green dashed line indicates the accuracy of prediction from using a $50 \mathrm{k}$ SNP array only. Results are based on cross-validation with 10,717 animals

was sometimes observed by including a larger number of SNPs (lower significant threshold) in $\mathbf{G}_{2}$ (Fig. 2; red dashed line), a more efficient use of the extra information might be achieved when a smaller number of preselected SNPs is included in SNP arrays.

Results from the GWAS based on imputed sequences revealed many strong signals across the genome for all traits with the most significant SNPs being found for CWT and BFT (results not shown). In general, the number of significant SNPs identified for CWT was largest $(10,892)$ with $\sim 8000$ significant SNPs identified for the other traits (Fig. 2).

Based on the results from the largest discovery dataset (4000 animals), a list of the candidate genes that are located close to the most significant SNPs with a high threshold value of $p<1.0 \mathrm{E}-05$ was identified (see Table 4). CWT is the trait with the largest number of genes identified, most of which are located on Bos taurus chromosome (BTA) 4, 10 and 14.

\section{Pre-selection of SNPs based on gene expression}

A large numbers of SNPs were pre-selected based on the results from gene expression (Table 5). A significance threshold of the $p$-value $<0.0032$ was set to include as many eQTL as possible since the SNPs in high LD were removed and because considering a stringent $p$-value could leave out important information. In total, 452,258 unique eQTL were detected across all ages (Table 5). We identified 76,018, 45,779, 38,742, 58,890, 354,530 eQTL at $8,12,18,24$, and 30 months of age, respectively, with on average 24,448 eQTL shared across all ages. After combining the lists of the eQTL for all ages and removing the SNPs in high LD $\left(\mathrm{r}^{2}>0.95\right), 130,748$ unique SNPs remained. The exact number of SNPs per reference-validation dataset is in Table 5. The number of SNPs that were pre-selected from the GSA results ranged from 19,000 to 36,000 SNPs after removing SNPs in high LD $\left(r^{2}>0.95\right)$, depending on the trait (Table 5).

\section{Comparison of prediction methods}

We compared different methods for genomic prediction using 10,717 animals as RV dataset (Fig. 3). Regardless of the method of preselecting SNPs, we observed no large differences between the methods evaluated. Comparing the achieved accuracies from adding pre-selected SNPs, the top SNPs from GWAS were the best source of information to improve the accuracy of prediction for carcass traits. For all traits, there were small improvements in the accuracies of prediction when using pre-selected SNPs 
Table 4 Genes located close to significant SNPs $(p<1.0 E-05)$ associated with carcass traits from GWAS on a discovery dataset of $\mathbf{4 0 0 0}$ animals

\begin{tabular}{|c|c|c|c|c|c|}
\hline Gene name (symbol) & Trait & SNP position (Chr:bp) & MAF & p-value & $\%$ \\
\hline ENSBTAG00000039810 & MS & 23:33569238 & 0.31 & $6.13 \mathrm{E}^{-06}$ & 3 \\
\hline N-ethylmaleimide-sensitive factor attachment protein, gamma (NAPG) & EMA & 24:42615958 & 0.32 & $8.29 \mathrm{E}^{-06}$ & 3 \\
\hline ENSBTAG00000033237 & EMA & 29:36927709 & 0.44 & $6.32 \mathrm{E}^{-06}$ & 3 \\
\hline Epidermal growth factor receptor pathway substrate 15 like 1 (EPS15L1) & $\mathrm{BFT}$ & 7:6484738 & 0.06 & $9.94 \mathrm{E}^{-06}$ & 2 \\
\hline SAM pointed domain containing ETS transcription factor (SPDEF) & BFT & 23:8537156 & 0.18 & $7.04 \mathrm{E}^{-06}$ & 2 \\
\hline HEPACAM family member 2 (HEPACAM2) & CWT & 4:10371904 & 0.11 & $1.75 \mathrm{E}^{-06}$ & 3 \\
\hline G protein subunit gamma transducin 1 (GNGT1) & CWT & 4:11059866 & 0.10 & $4.66 \mathrm{E}^{-08}$ & 4 \\
\hline ENSBTAG00000035660 & CWT & 4:13589367 & 0.05 & $9.43 \mathrm{E}^{-08}$ & 4 \\
\hline Fidgetin like 1 (FIGNL1) & CWT & 4:5373926 & 0.10 & $3.15 \mathrm{E}^{-07}$ & 4 \\
\hline ENSBTAG00000018039 & CWT & 10:95431561 & 0.47 & $5.99 \mathrm{E}^{-06}$ & 3 \\
\hline Coiled-coil-helix-coiled-coil-helix domain containing 7 (CHCHD7) & CWT & 14:25059742 & 0.34 & $3.23 \mathrm{E}^{-10}$ & 6 \\
\hline Ribosomal protein L39 (RPL39) & CWT & 14:26181231 & 0.17 & $8.75 \mathrm{E}^{-09}$ & 5 \\
\hline UBX domain protein $2 B(U B X N 2 B)$ & CWT & 14:26303702 & 0.28 & $3.41 \mathrm{E}^{-06}$ & 3 \\
\hline Thymocyte selection associated high mobility group box (TOX) & CWT & 14:26941314 & 0.18 & $1.08 \mathrm{E}^{-06}$ & 4 \\
\hline ENSBTAG00000014045 & CWT & 14:29678929 & 0.24 & $8.54 \mathrm{E}^{-06}$ & 3 \\
\hline KDEL endoplasmic reticulum protein retention receptor 2 (KDELR2) & CWT & 25:38887854 & 0.19 & $3.07 \mathrm{E}^{-06}$ & 3 \\
\hline
\end{tabular}

Chr chromosome, bp base pairs, MAF minor allele frequency; \% percentage of variance explained by the genotype

Table 5 Number of SNPs used in the genomic prediction, pre-selected from gene expression analysis and after pruning (and the SNPs remaining in the $50 \mathrm{k}$-G50adj in brackets) in the various RV subsets

\begin{tabular}{lccrrrr}
\hline & Genes & SNPs & \multicolumn{2}{l}{ Reference-validation datasets } & & \\
\cline { 3 - 6 } & & & $\mathbf{1 2 , 7 1 7}$ & $\mathbf{1 1 , 7 1 7}$ & $\mathbf{1 0 , 7 1 7}$ & $\mathbf{9 7 1 7}$ \\
\hline eQTL & 10,224 & 452,258 & $130,750(40,129)$ & $130,251(40,135)$ & $130,080(40,130)$ & $130,582(40,124)$ \\
GSA $_{\text {MS }}$ & 473 & 98,099 & $23,398(40,774)$ & $23,338(40,776)$ & $23,280(40,778)$ & $23,366(40,769)$ \\
GSA $_{\text {EMA }}$ & 367 & 76,585 & $19,852(40,805)$ & $19,799(40,803)$ & $19,759(40,804)$ & $19,817(40,806)$ \\
GSA $_{\text {CWT }}$ & 440 & 104,512 & $24,361(40,740)$ & $24,298(40,743)$ & $24,268(40,744)$ & $24,327(40,739)$ \\
GSA $_{\text {BFT }}$ & 810 & 146,958 & $36,219(40,661)$ & $36,116(40,658)$ & $36,056(40,633)$ & $36,168(40,658)$ \\
\hline
\end{tabular}

GSA gene expression significantly associated, BFT back fat thickness, EMA eye muscle area, CWT carcass weight, and MS marbling score

from the eQTL (Fig. 3) but no benefit was observed from using SNPs from GSA (not shown).

Results from wGBLUP and GBLUP were compared for both Models 2 and 3 (using either $50 \mathrm{k}$ or $50 \mathrm{k}$ and top-SNPs from the GWAS discovery set of 3000 animals (50 k_GWAS; Table 6). Accuracies of prediction were similar regardless of the number of iteration rounds used, although accuracy of prediction tended to be higher after two or three iteration rounds. When using the $50 \mathrm{k} \mathrm{panel}$, wGBLUP showed a small improvement in accuracy over the standard GBLUP method of 0.01 for BFT, whereas for MS and EMA the accuracy was not improved. The use of the $50 \mathrm{k}$ _GWAS SNP panel in wGBLUP improved the accuracy of prediction slightly more, i.e. from 0.01 to 0.05 (Table 6).

For MS, the use of top SNPs increased the accuracy of prediction by 0.05 for GBLUP-GWAS, and by 0.02 for wGBLUP-GWAS and BayesR-GWAS (Fig. 3). Preselected SNPs from eQTL and GWAS improved the prediction for EMA in all models compared with the $50 \mathrm{k}$ baseline (Fig. 3). The largest improvement in prediction for CWT was achieved with BayesR-GWAS and GBLUPGWAS by 0.04 and 0.03 , respectively. For BFT, the largest improvement was observed when the wGBLUP-GWAS and BayesR-GWAS models were implemented with an increase of 0.06 (Fig. 3). For MS, BFT, and CWT, the addition of SNPs from GWAS increased the accuracy of prediction compared to using SNPs from eQTL whereas using eQTL SNPs proved to be the same or better than the GWAS SNPs for the prediction of EMA.

We found no accuracy increase for CWT and BFT when comparing results from using $\mathbf{G}_{1}$ based on the $50 \mathrm{k}$ standard array and using a second $\mathbf{G}_{2}$ from SNPs that 

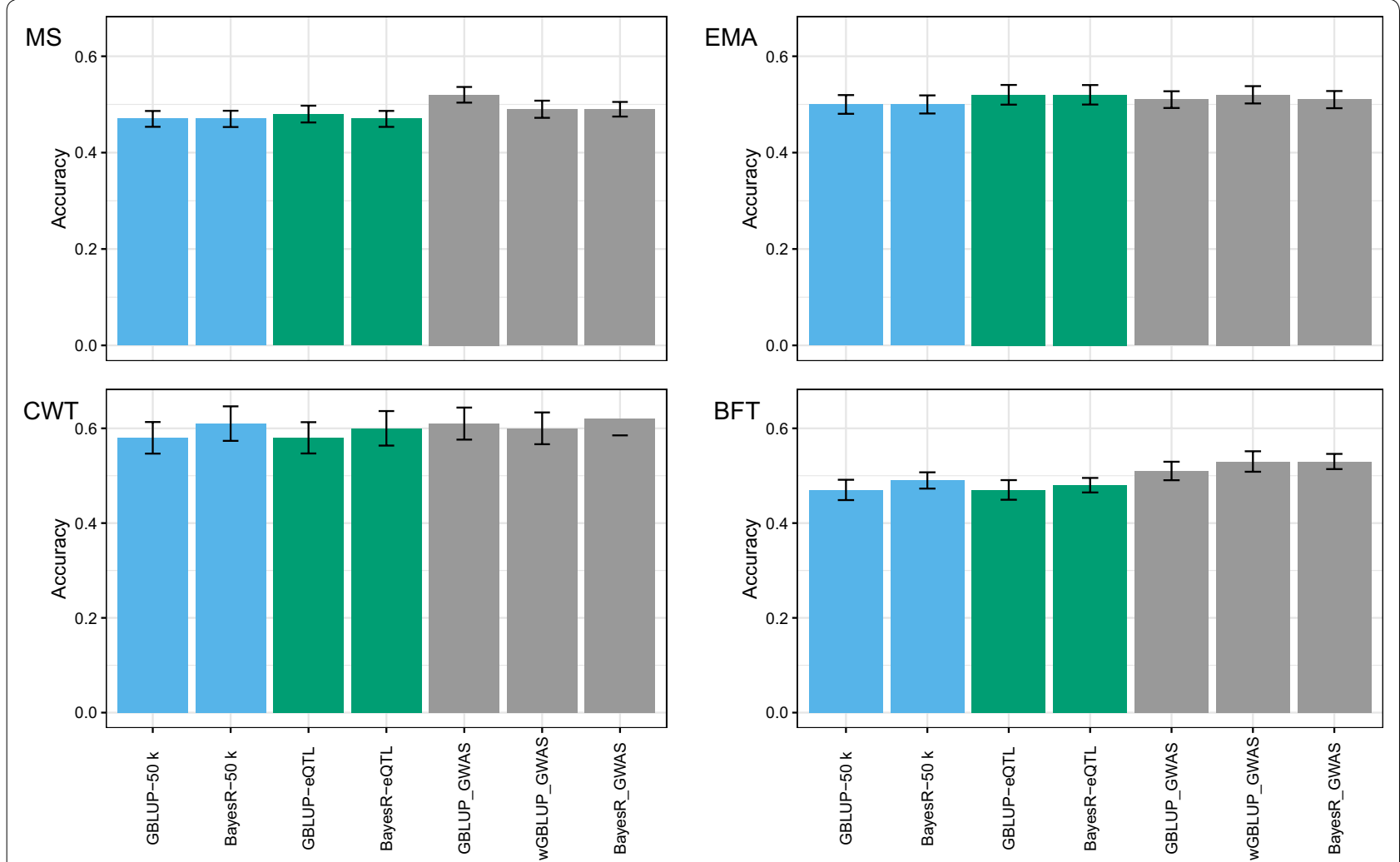

Fig. 3 Accuracy of genomic prediction of breeding value for carcass traits marbling score (MS), eye muscle area (EMA), carcass weight (CWT) and back fat thickness (BFT) comparing GBLUP, wGBLUP, and BayesR models using three SNP sets: (1) standard 50 k array (blue bars); (2) 50 k and eQTL-SNPs (green bars) and (3) $50 \mathrm{k}$ and GWAS top-SNPs (grey bars). All analysis use cross-validation (CV) with 10,717 animals. Vertical lines indicate the empirical standard error for each CV result

were selected based on eQTL, but accuracies of prediction for EMA improved by 0.02 (Fig. 3).
The biases observed for EMA and BFT were lower than for MS and CWT (Fig. 4).

Table 6 Accuracy of prediction of breeding value for carcass traits (SE) in consecutive iterations of wGBLUP with $50 \mathrm{k}$ and a combination of $50 \mathrm{k}$ and top-SNPs from GWAS (50 k_GWAS)

\begin{tabular}{|c|c|c|c|c|c|}
\hline Model & Iteration & MS & EMA & CWT & BFT \\
\hline 50 k (GBLUP) & 1 & $0.47(0.02)$ & $0.51(0.02)$ & $0.58(0.03)$ & $0.48(0.02)$ \\
\hline \multirow[t]{4}{*}{50 k (wGBLUP) } & 2 & $0.47(0.02)$ & $0.51(0.02)$ & $0.58(0.03)$ & $0.48(0.02)$ \\
\hline & 3 & $0.47(0.02)$ & $0.50(0.02)$ & $0.59(0.03)$ & $0.49(0.02)$ \\
\hline & 4 & $0.47(0.02)$ & $0.49(0.02)$ & $0.58(0.03)$ & $0.50(0.02)$ \\
\hline & 5 & $0.46(0.02)$ & $0.48(0.02)$ & $0.58(0.03)$ & $0.49(0.02)$ \\
\hline \multirow[t]{4}{*}{50 k_GWAS (wGBLUP) } & 2 & $0.49(0.02)$ & $0.52(0.02)$ & $0.60(0.03)$ & $0.52(0.02)$ \\
\hline & 3 & $0.49(0.02)$ & $0.51(0.02)$ & $0.60(0.03)$ & $0.53(0.02)$ \\
\hline & 4 & $0.49(0.02)$ & $0.51(0.02)$ & $0.60(0.03)$ & $0.53(0.02)$ \\
\hline & 5 & $0.48(0.02)$ & $0.50(0.02)$ & $0.59(0.03)$ & $0.52(0.02)$ \\
\hline
\end{tabular}

BFT back fat thickness, EMA eye muscle area, CWT carcass weight, and MS marbling score

Genomic predictions for all carcass traits showed some bias but the bias observed with wGBLUP was generally much higher than that with the other methods (Fig. 4).

\section{Discussion}

The aim of this study was to assess the accuracy of prediction for carcass traits in Hanwoo cattle when using GBLUP, wGBLUP, and a BayesR model, and the potential 

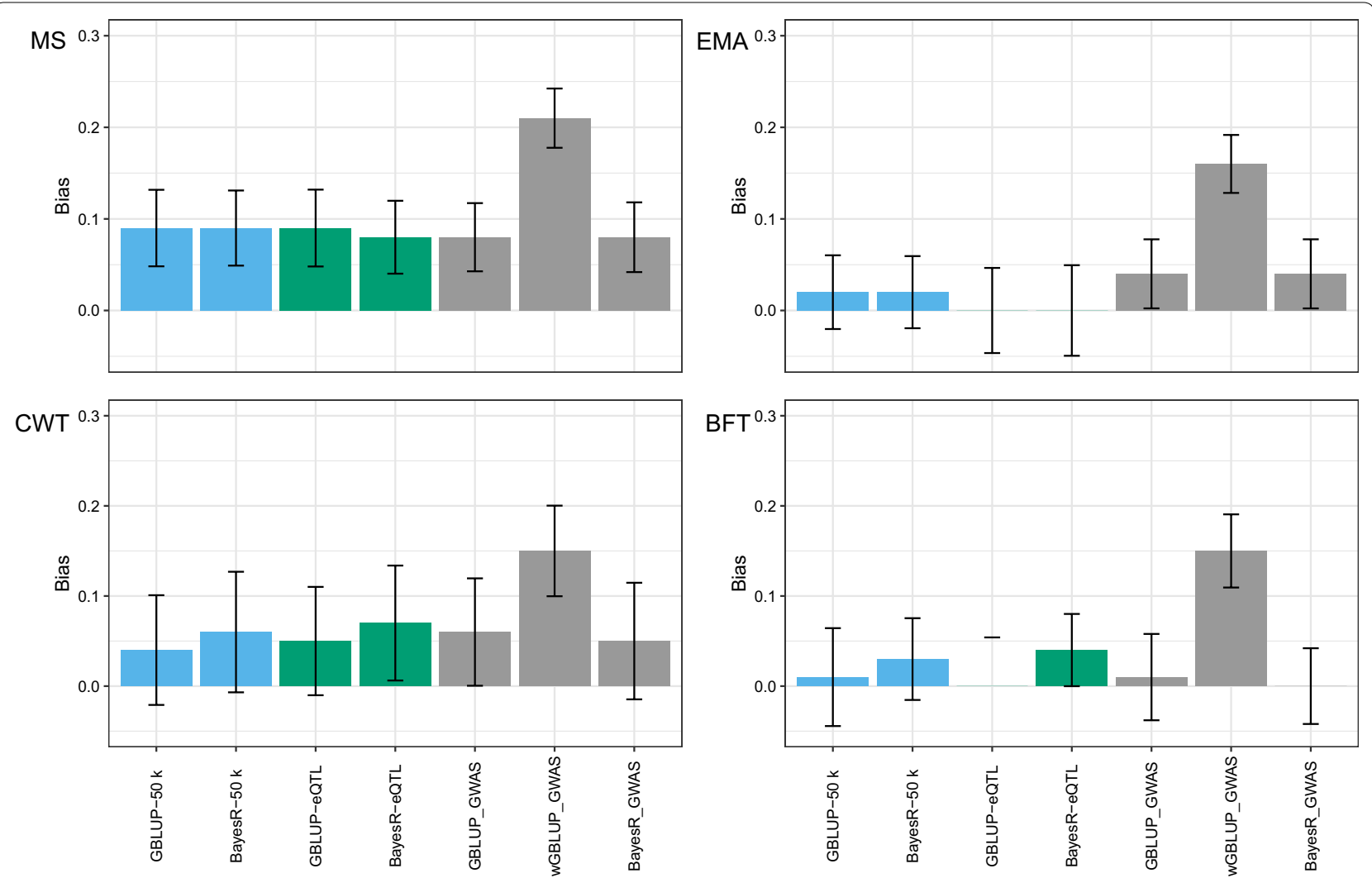

Fig. 4 Bias of genomic prediction of breeding value for carcass traits marbling score (MS), eye muscle area (EMA), carcass weight (CWT) and back fat thickness (BFT) comparing GBLUP, wGBLUP, and BayesR models using three SNP sets: (1) standard $50 \mathrm{k}$ array (blue bars); (2) $50 \mathrm{k}$ and eQTL-SNPs (green bars) and (3) $50 \mathrm{k}$ and GWAS top-SNPs (grey bars). All analyses use cross-validation (CV) with 10,717 animals. Vertical lines indicate the empirical standard error for each CV result

gain from using pre-selected SNPs from a GWAS on imputed sequence data as well as from gene expression information. We found that pre-selected SNPs from GWAS improved the accuracy of prediction slightly, whereas gene expression SNPs that are located in genes, the expression of which is associated with phenotypic differences in carcass traits, were less useful to improve prediction accuracy.

During the last decade, GBLUP was applied in many breeding programs for plants and animals and resulted in increased rates of genetic gain [40, 41]. To date, genomic selection is not commonly applied in Hanwoo cattle breeding programs to improve carcass traits. In this study, first we established a baseline accuracy of prediction for Hanwoo cattle, using the standard $50 \mathrm{k}$ SNP array and a ten-fold cross validation, and compared the accuracy obtained from $\mathrm{k}$-means cross-validation with random selection. Higher accuracies (an increase ranging from 0.04 to 0.07 , depending on the trait) were observed with random CV. Since the k-means CV strategy is based on clustering according to the relatedness between samples, it is less likely that animals in the validation set have related animals in the reference training set. Therefore, relatedness between training and test animals has a fairly large effect on accuracy of prediction, as was previously pointed out in the literature [42]. We also observed that, with a random CV strategy, the benefit of using top-SNPs was smaller (results not shown). The benefit of including prioritized SNPs for genomic prediction is larger when the genomic relationships between the reference and validation sets are lower [17]. Since the predictive SNPs were selected based on an association with the phenotype in relatively unrelated individuals, their contribution to accuracy of prediction should be less affected by relatedness. Similarly, we reported an improvement in accuracy of prediction when a larger reference population was used, as previously reported [43, 44], which also reduces the marginal value of using more predictive SNPs. Thus, the benefit of using more predictive SNPs is most likely greater when the baseline accuracy is lower and when the individuals being predicted have a lower relatedness to the reference population.

To select the GWAS discovery dataset, the more genetically diverse animals were chosen, resulting in a lower 
degree of LD in the discovery set and therefore a higher resolution of the QTL regions identified. Previous studies have shown that SNPs that are located near QTL detected in multibreed datasets tend to be more useful in improving accuracy of prediction $[8,45]$, and this is expected to be due to the lower LD that exists in multibreed datasets. In such a case, detection of QTL would require a higher marker density, but fewer markers would be in LD with the QTL across a set of more diverse individuals, hence allowing more precise mapping of the QTL. While we did not use a multi-breed discovery set, a greater diversity in the discovery set of a single breed might also prove to be more useful.

The use of more individuals for the GWAS is expected to improve the identification of associated SNPs. The use of imputed sequence genotype data increased the number of detected QTL and their $p$-values compared with $50 \mathrm{k}$ (results not shown) especially for traits that seem to behave more highly polygenic (MS, EMA, and BFT). However, a larger number of variants might also lead to more false positives. The amount of overlap in QTL between the various discovery sets was small in our study, which suggests that most of the significant SNPs might not be real QTL and could partly explain the limited increase in accuracy from using the top SNPs. Although the size of the dataset used in this study was relatively large, at least for beef cattle, it is clear that even larger datasets are needed in GWAS to successfully identify SNPs that will increase accuracy of prediction.

There is no consensus in the literature about the threshold that should be used for selecting top SNPs for the purpose of improving prediction. In this study, we tried to keep a balance between the number of SNPs used and the increase in the accuracy of prediction. In general, the use of larger discovery datasets improved the identification of informative SNPs and these SNPs did increase the accuracy of prediction for all the traits. The top-SNPs that increased the accuracy of prediction were identified using a low significance threshold ( $p$-value $<0.0032)$, and after filtering for LD $\left(r^{2}>0.95\right)$, less than $30 \%$ of the SNPs were kept (Table 5).

The use of the larger dataset for GWAS did allow to identify QTL regions associated with carcass traits located on the NAPG, EPS15L1, SPDEF, HEPACAM2, GNGT1, FIGNL1, CHCHD7, RPL39, UBXN2B, TOX, and KDELR2 genes among other non-annotated genes (Table 4). In a previous study in Hanwoo cattle, BTA4, 6, and 14 were significantly associated with CWT and EMA [14]. The EPS15L1 gene is an essential component of the endocytic pathway [46]. The SPDEF gene is known to be associated with BFT in pigs [47]. The HEPACAM2 gene was previously identified in a QTL region for mid-test metabolic weight (MMWT) in SimAngus on BTA4 [48].
The $\mathrm{CHCHD} 7$ gene on BTA14 is associated with CWT in the Hanwoo breed [11], with knuckle, biceps and shank trait in Chinese Simmental cattle [49], with carcass weight in Japanese Black steers [50], with back fat thickness and rump fat thickness in the Nellore breed [51], and with fat thickness and intramuscular fat in composite cattle [52]. The $U B X N 2 B$ gene is associated with MMWT in SimAngus [48], and with carcass weight, carcass fat, and carcass conformation in Simmental [53]. The TOX gene is located within the QTL that is associated with CWT and EMA in Hanwoo cattle [14], and with residual feed intake and MMWT [48]. The expression of KDELR2 is upregulated in response to endoplasmic reticulum stress to stabilize exocytosis [54] but no studies have reported an association of this gene with CWT in cattle.

Although our GWAS results revealed some SNPs that are located on well-known genes for each trait, especially for CWT (Table 4), larger datasets are needed to refine the regions that are associated with EMA, MS, and BFT. We observed that, after pruning, the SNPs were not always located close to the polymorphisms within informative functional genes that help to understand the biological nature of the traits. Nevertheless, overall these markers explain some of the genetic variation associated with the traits in this study and had a small to modest effect on the accuracy of predicting GBV. Hanwoo cattle have a small effective population size, which has an increasing effect on the LD between SNPs and causal variants, but this argument holds for SNPs in the standard $50 \mathrm{k}$ array as well as for SNPs selected from the sequence data. Further evaluation of optimal ways of selecting SNPs from a discovery population and SNP filtering methods based on LD and allele frequency are recommended to optimize the identification of the most suitable QTL for improving genomic selection.

Applying an additional G matrix for the top SNPs in the GBLUP model effectively gives more weight to the top-SNPs, since few SNPs share a relatively large variance component to calculate the GBV. Our results show that this model is more effective to improve the accuracies for all carcass traits in Hanwoo cattle (Fig. 4), even for polygenic traits such as MS and EMA. The observed improvement in accuracy of prediction suggests that these pre-selected SNPs indeed contain some additional information compared to neutral markers on the standard $50 \mathrm{k} \mathrm{SNP}$ array. Brøndum et al. [55] reported that the use of top-SNPs together with the $50 \mathrm{k}$ SNP chip in a GBLUP model increased the accuracy of prediction by $5 \%$ for production traits in dairy cattle. In a study based on sheep data, the use of two $\mathbf{G}$ matrices (50 k and significant variants from the GWAS) to estimate GBV led to an increase in accuracy of prediction by on average 6.2 and $4.1 \%$ for purebred and crossbred sheep, respectively [8]. 
In previous studies, the advantage of BayesR over GBLUP was more pronounced for traits that are influenced by few QTL with moderate to large effects. The BayesR method resulted in a higher accuracy (on average 0.05 across traits) than GBLUP for milk yield and fat yield but there was no difference in the accuracy of prediction for protein yield [56]. Similarly, our results for CWT and BFT, for which more significant QTL regions were found, show that the use of BayesR-50 k increased the prediction accuracy by 0.03 and 0.02 compared to that of GBLUP$50 \mathrm{k}$ (Fig. 3), whereas there was no improvement for the other traits. In addition, a larger improvement in accuracy of prediction was observed for BFT (0.06) and CWT (0.04) when BayesR with pre-selected SNPs (BayesRGWAS) was used. In sheep, the use of top SNPs in combination with BayesR increased the accuracy by 0.09 in Merino and 0.06 in crossbreds [8].

The use of top SNPs in wGBLUP also improved the accuracy but only by 0.02 for MS, 0.01 for EMA, 0.02 for CWT, and 0.06 for BFT (Fig. 3). However, the wGBLUP-GWAS method also tended to increase the bias of the prediction quite severely, by 0.11 to 0.14 , depending on the trait (Fig. 4). After reaching the fifth iteration, a higher bias was observed. Similarly, Lopez et al. [37] reported a reduction in the regression coefficient (increased bias) at each additional iteration. This increase was expected since we assigned more weight to SNPs with a large effect in the training population but these SNP effects tended to be overestimated. An increase in bias was also reported by [57] in a single-step wGBLUP strategy.

The differences observed in the performance of the methods depend strongly on the genetic structure of each trait. For polygenic traits such as MS, we observed that the performance of GBLUP-GWAS was superior to that of Bayes-GWAS. In a simulated dataset, the use of BayesR for highly polygenic traits resulted in more variable heritability estimates and slightly lower accuracies of prediction compared to GBLUP [39]. A simulation study testing the wGBLUP method showed that a trait with a larger number of QTL has a smaller increase in accuracy (0.05) than a trait with an only small number of QTL whereas the accuracy could increase by 0.1 when the weighting SNPs effects were considered in the wGBLUP model [58].

In our study, we used imputed SNPs at the sequence level and these imputations were derived from other imputed sequence data. Imputed sequence data could potentially include errors and these errors would be further propagated when the data are used as a reference for further imputation of other samples. We used whole-genome sequence SNPs that had been imputed by a Minimac3 accuracy higher than 0.4 , but the accuracy of imputation might be inflated if imputed genotypes were used as a reference. It has been reported that imputation errors [59] and especially errors in the imputation to whole-genome sequences data could lower the accuracy of prediction by 0.01 to 0.03 [3]. Moreover, accuracy of imputation tends to be lower for SNPs with a lower MAF [60]. The use of larger and more diverse reference populations as well as a larger sample of individuals in the reference set with real sequence data should be considered for imputation purposes to better capture rare markers [61].

For complex traits, the use of more biological information (protein, metabolite, etc.) has been suggested to identify genomic features that are enriched for causal variants, and these were shown to increase the accuracy of prediction slightly by using the SNPs located in the best-performing gene ontology terms information [18]. In our study, no improvement was observed in the accuracy of prediction of any trait using the SNPs that were selected from GSA, although a significantly larger number of markers were included in the $\mathbf{G}_{2}$ (from $\sim 19,000$ to 36,000 SNPs) compared to using only the $50 \mathrm{k}$ SNP panel. It is possible that SNPs located in these genes have a low level of heterozygosity and therefore only a small part of the variance of the phenotype is explained. It is also important to point out that only 45 animals were used in the gene expression study and this is a limited number of samples for identifying the GSA genes. Moreover, these gene expression patterns might change with tissue and development stage of sampling. The expression profile (GSA) from the longissimus dorsi muscle may have a small effect on traits, such as CWT and BFT. In another study, the use of SNPs from a high-density panel (800 k) that were located in exons did not improve the accuracy of prediction over the $50 \mathrm{k}$ or $800 \mathrm{k}$ SNP chips when analyzing milk traits in a model that included these SNPs [56].

Simulation studies have shown that using the actual causal variants could significantly increase the accuracy of prediction [62], but this increase does depend on the size of the QTL detected [42]. Wang et al. [63] reported that the use of true QTL in combination with non-QTLmarkers decreased the accuracy of prediction. Therefore, to what extent are the selected SNPs actual causal variants, or at least in very high LD with causal variants is important for their usefulness for prediction. GWAS studies based on sequence data are often still underpowered, given the large multiple testing challenge, but at least larger GWAS datasets might be able to better select the SNPs that can explain and predict genetic variation. While expression studies can help find functional genes relevant to phenotypes of interest, fundamentally they do not detect SNPs based on their ability to explain variation 
in those phenotypes. Moreover, these studies are usually much smaller in size and depend largely on the timing and type of tissue sampled. Therefore, expression studies might be useful to support GWAS to detect more predictive SNPs, but when used alone, they have a lower chance of being successful than GWAS studies for that purpose.

\section{Conclusions}

For all traits, the accuracy of prediction slightly improved by pre-selecting SNPs from GWAS, whereas only a slight and non-significant improvement was obtained when using eQTL SNPs. The use of SNPs selected from GSA did not lead to an improvement in the genomic prediction of carcass traits compared to the standard $50 \mathrm{k}$ SNP panel. The performance of each method for estimating GBV is trait-specific and likely depends on the genetic architecture of each trait. The GBLUP-GWAS method reached a higher accuracy of prediction for MS, whereas the BayesR method was better for CWT and BFT. In future studies, the use of a larger discovery dataset for GWAS will help to improve the identification of more QTL regions and therefore will potentially increase the accuracy of prediction for carcass traits especially for traits, such as EMA and MS. In addition, larger and more targeted gene expression studies should be used and combined with GWAS to show whether these studies have potential to provide more predictive SNPs.

\section{Supplementary information}

Supplementary information accompanies this paper at https://doi. org/10.1186/s12711-020-00574-2.

Additional file 1: Figure S1. Venn diagram with the number of samples that overlap between four sizes of the discovery dataset (D; from 1000 to 4000 animals). Marbling score (MS), eye muscle area (EMA), carcass weight (CWT), and back fat thickness (BFT).

\section{Acknowledgements}

The authors acknowledge the Korean Institute of Animal Products Quality Evaluation (KAPE) for providing the phenotypic data.

\section{Authors' contributions}

DL and SHL obtained the genotype and phenotype data and with SD, obtained the RNA-Seq data. KYC designed the gene expression experiment and obtained the samples and DL, WCP, and JEP analyzed the RNA-Seq data. SDLHS and JHJVDW designed the analysis strategy and the analysis was overseen by NM, SHL and DL. SDLHS performed the imputation, gene expression (GSA, eQTL) analysis, and GWAS. SDLHS and BL both ran the analytical models for genomic prediction. SDLHS wrote the first draft of the manuscript. JHJVDW edited and improved the manuscript. All authors read and approved the final manuscript.

\section{Funding}

The authors gratefully acknowledge funding from the BIOGREEN project (No. PJ012611). Bryan Irvine Lopez was supported by the 2020 RDA Research Associate Fellowship Program of the National Institute of Animal Science, Rural Development Administration, Republic of Korea.
Availability of data and materials

Not applicable.

Ethics approval and consent to participate

Not applicable.

\section{Consent for publication}

Not applicable.

\section{Competing interests}

The authors declare that they have no competing interests.

\section{Author details}

${ }^{1}$ School of Environmental and Rural Science, University of New England, Armidale NSW 2351, Australia. ${ }^{2}$ Animal Genomics and Bioinformatics Division, National Institute of Animal Science, Rural Development Administration, Wanju 55365, Republic of Korea. ${ }^{3}$ Department of Beef Science, Korea National College of Agriculture and Fisheries, Jeonju, Republic of Korea. ${ }^{4}$ Division of Animal and Dairy Science, Chungnam National University, Deajeon 34148, Republic of Korea. ${ }^{5}$ The Animal Molecular Genetics and Breeding Centre, Jeonbuk National University, Jeonju 54896, Republic of Korea.

Received: 27 May 2020 Accepted: 18 September 2020

Published online: 29 September 2020

\section{References}

1. Chung KY, Lee SH, Cho SH, Kwon EG, Lee JH. Current situation and future prospects for beef production in South Korea-A review. Asian-Australas J Anim Sci. 2018;31:951-60.

2. Mehrban H, Lee DH, Moradi MH, IICho C, Naserkheil M, Ibáñez-Escriche N. Predictive performance of genomic selection methods for carcass traits in Hanwoo beef cattle: impacts of the genetic architecture. Genet Sel Evol. 2017:49:1.

3. van Binsbergen R, Calus MPL, Bink MCAM, van Eeuwijk FA, Schrooten C, Veerkamp RF. Genomic prediction using imputed whole-genome sequence data in Holstein Friesian cattle. Genet Sel Evol. 2015;47:71.

4. Veerkamp RF, Bouwman AC, Schrooten C, Calus MPL. Genomic prediction using preselected DNA variants from a GWAS with whole-genome sequence data in Holstein-Friesian cattle. Genet Sel Evol. 2016;48:95.

5. Raymond B, Bouwman AC, Schrooten C, Houwing-Duistermaat J, Veerkamp RF. Utility of whole-genome sequence data for across-breed genomic prediction. Genet Sel Evol. 2018;50:27.

6. VanRaden PM, Tooker ME, O'Connell JR, Cole JB, Bickhart DM. Selecting sequence variants to improve genomic predictions for dairy cattle. Genet Sel Evol. 2017:49:32.

7. Raven LA, Cocks BG, Kemper KE, Chamberlain AJ, Vander Jagt CJ, Goddard ME, et al. Targeted imputation of sequence variants and gene expression profiling identifies twelve candidate genes associated with lactation volume, composition and calving interval in dairy cattle. Mammal Genome. 2016;27:81-97.

8. Moghaddar N, Khansefid M, van der Werf JHJ, Bolormaa S, Duijvesteijn N, Clark S, et al. Genomic prediction based on selected variants from imputed whole-genome sequence data in Australian sheep populations. Genet Sel Evol. 2019;51:72.

9. Lee SH, van der Werf JHJ, Lee SH, Park EW, Gondro C, Yoon D, et al Genome wide QTL mapping to identify candidate genes for carcass traits in Hanwoo (Korean Cattle). Genes Genomics. 2012;34:43-9.

10. Lee $\mathrm{SH}$, Choi BH, Cho SH, Lim D, Choi TJ, Park BH, et al. Genome-wide association study identifies three loci for intramuscular fat in Hanwoo (Korean cattle). Livest Sci. 2014;165:27-32.

11. Lee SH, Choi BH, Lim D, Gondro C, Cho YM, Dang CG, et al. Genome-wide association study identifies major loci for carcass weight on BTA14 in Hanwoo (Korean cattle). PLoS One. 2013;8:e74677.

12. Lee YM, Han CM, Li Y, Lee JJ, Kim LH, Kim JH, et al. A whole genome association study to detect single nucleotide polymorphisms for carcass traits in Hanwoo populations. Asian-Australas J Anim Sci. 2010;23:417-24.

13. Kim Y, Ryu J, Woo J, Kim JB, Kim CY, Lee C. Genomewide association study reveals five nucleotide sequence variants for carcass traits in beef cattle. Anim Genet. 2011;42:361-5. 
14. Bhuiyan M, Lim D, Park M, Lee S, Kim Y, Gondro C, et al. Functional partitioning of genomic variance and genome-wide association study for carcass traits in Korean Hanwoo cattle using imputed sequence level SNP data. Front Genet. 2018;9:217.

15. Dang CG, Cho SH, Sharma A, Kim HC, Jeon GJ, Yeon SH, et al. Genomewide association study for Warner-Bratzler shear force and sensory traits in Hanwoo (Korean cattle). Asian-Australas J Anim Sci. 2014;27:1328-35.

16. Strucken EM, Al-Mamun HA, de las Heras-Saldana S, Bedhane MN, Lim D, Park $B$, et al. Finding the marble-The polygenic architecture of intramuscular fat. J Anim Breed Genomics. 2017;1:69-76.

17. MacLeod IM, Bowman PJ, Vander Jagt CJ, Haile-Mariam M, Kemper KE, Chamberlain AJ, et al. Exploiting biological priors and sequence variants enhances QTL discovery and genomic prediction of complex traits. BMC Genomics. 2016;17:144.

18. Fang L, Sahana G, Ma P, Su G, Yu Y, Zhang S, et al. Use of biological priors enhances understanding of genetic architecture and genomic prediction of complex traits within and between dairy cattle breeds. BMC Genomics. 2017;18:604.

19. Xiang R, Berg I, MacLeod IM, Hayes BJ, Prowse-Wilkins CP, Wang M, et al. Quantifying the contribution of sequence variants with regulatory and evolutionary significance to 34 bovine complex traits. Proc Natl Acad Sci USA. 2019;116:19398-408.

20. Lee SH, Cho YM, Lee SH, Kim BS, Kim NK, Choy YH, et al. Identification of marbling-related candidate genes in M. longissimus dorsi of high-and low marbled Hanwoo (Korean Native Cattle) steers. BMB Rep. 2008;41:846-51

21. Lim D, Chai HH, Lee SH, Cho YM, Choi JW, Kim NK. Gene expression patterns associated with peroxisome proliferator-activated receptor (PPAR) signaling in the longissimus dorsi of Hanwoo (Korean Cattle). AsianAustralas J Anim Sci. 2015;28:1075-83.

22. Lim D, Kim NK, Park HS, Lee SH, Cho YM, Oh SJ, et al. Identification of candidate genes related to bovine marbling using protein-protein interaction networks. Int J Biol Sci. 2011;7:992-1002.

23. Lim D, Kim NK, Lee SH, Park HS, Cho YM, Chai HH, et al. Characterization of genes for beef marbling based on applying gene coexpression network. Int J Genomics. 2014;2014:708562.

24. Lee SH, Gondro C, van der Werf JHJ, Kim NK, Lim DJ, Park EW, et al. Use of a bovine genome array to identify new biological pathways for beef marbling in Hanwoo (Korean Cattle). BMC Genomics. 2010;11:623.

25. Andrews S. FastQC: a quality control tool for high throughput sequence data. 2010. https://www.bioinformatics.babraham.ac.uk/projects/fastqc/ Accessed 15 Sept 2020.

26. Bolger AM, Lohse M, Usadel B. Trimmomatic: a flexible trimmer for Illumina sequence data. Bioinformatics. 2014;30:2114-20.

27. Kim D, Langmead B, Salzberg SL. HISAT: a fast spliced aligner with low memory requirements. Nat Methods. 2015;12:357-60.

28. Liao Y, Smyth GK, Shi W. featureCounts: an efficient general purpose program for assigning sequence reads to genomic features. Bioinformatics. 2014;30:923-30.

29. Loh PR, Palamara PF, Price AL. Fast and accurate long-range phasing in a UK Biobank cohort. Nat Genet. 2016;48:811-6.

30. Das S, Forer L, Schönherr S, Sidore C, Locke AE, Kwong A, et al. Nextgeneration genotype imputation service and methods. Nat Genet. 2016:48:1284-7.

31. Chang CC, Chow CC, Tellier LC, Vattikuti S, Purcell SM, Lee JJ. Secondgeneration PLINK: rising to the challenge of larger and richer datasets. Gigascience. 2015;4:7.

32. Gilmour AR, Gogel BJ, Cullis BR, Welham SJ, Thompson R. ASReml user guide release 4.1 structural specification. Hemel hempstead: VSN international ltd. 2015.

33. Yang J, Lee SH, Goddard ME, Visscher PM. GCTA: a tool for genome-wide complex trait analysis. Am J Hum Genet. 2011;88:76-82.

34. Shabalin AA. Matrix eQTL: ultra fast eQTL analysis via large matrix operations. Bioinformatics. 2012;28:1353-8.

35. Lee SH, Van der Werf JHJ. MTG2: an efficient algorithm for multivariate linear mixed model analysis based on genomic information. Bioinformatics. 2016;32:1420-2.

36. Wang H, Misztal I, Aguilar I, Legarra A, Muir WM. Genome-wide association mapping including phenotypes from relatives without genotypes. Genet Res. 2012;94:73-83.
37. Lopez BI, Lee SH, Park JE, Shin DH, Oh JD, de las Heras-Saldana S, et al. Weighted genomic best linear unbiased prediction for carcass traits in Hanwoo cattle. Genes. 2019;10:1019.

38. VanRaden PM. Efficient methods to compute genomic predictions. J Dairy Sci. 2008;91:4414-23.

39. Moser G, Lee SH, Hayes BJ, Goddard ME, Wray NR, Visscher PM. Simultaneous discovery, estimation and prediction analysis of complex traits using a Bayesian mixture model. PLoS Genet. 2015;11:e1004969.

40. Daetwyler HD, Swan AA, van der Werf JHJ, Hayes BJ. Accuracy of pedigree and genomic predictions of carcass and novel meat quality traits in multi-breed sheep data assessed by cross-validation. Genet Sel Evol. 2012:44:33.

41. Chen $L$, Schenkel F, Vinsky M, Crews DH Jr, Li C. Accuracy of predicting genomic breeding values for residual feed intake in Angus and Charolais beef cattle. J Anim Sci. 2013;91:4669-78.

42. Clark SA, Hickey JM, van der Werf JHJ. Different models of genetic variation and their effect on genomic evaluation. Genet Sel Evol. 2011;43:18.

43. Bolormaa S, Pryce JE, Kemper K, Savin K, Hayes BJ, Barendse W, et al. Accuracy of prediction of genomic breeding values for residual feed intake and carcass and meat quality traits in Bos taurus, Bos indicus, and composite beef cattle. J Anim Sci. 2013;91:3088-104.

44. Boddhireddy P, Kelly M, Northcutt S, Prayaga KC, Rumph J, DeNise S. Genomic predictions in Angus cattle: comparisons of sample size, response variables, and clustering methods for cross-validation. J Anim Sci. 2014;92:485-97.

45. Saatchi M, Beever JE, Decker JE, Faulkner DB, Freetly HC, Hansen SL, et al. QTLs associated with dry matter intake, metabolic mid-test weight, growth and feed efficiency have little overlap across 4 beef cattle studies. BMC Genomics. 2014;15:1004.

46. Carbone R, Fré S, lannolo G, Belleudi F, Mancini P, Pelicci PG, et al. eps15 and eps15R are essential components of the endocytic pathway. Cancer Res. 1997;57:5498-504.

47. Lee YS, Shin D. Genome-wide association studies associated with backfat thickness in Landrace and Yorkshire pigs. Genomics Inform. 2018;16:59-64.

48. Seabury CM, Oldeschulte DL, Saatchi M, Beever JE, Decker JE, Halley YA, et al. Genome-wide association study for feed efficiency and growth traits in US beef cattle. BMC Genomics. 2017;18:386.

49. Song Y, Xu L, Chen Y, Zhang L, Gao H, Zhu B, et al. Genome-wide association study reveals the PLAG1 gene for knuckle, biceps and shank weight in Simmental beef cattle. PLoS One. 2016;11:e0168316.

50. Nishimura S, Watanabe T, Mizoshita K, Tatsuda K, Fujita T, Watanabe N, et al. Genome-wide association study identified three major QTL for carcass weight including the PLAG1-CHCHD7 QTN for stature in Japanese Black cattle. BMC Genet. 2012;13:40.

51. de Oliveira Silva RM, Stafuzza NB, de Oliveira Fragomeni B, de Camargo GMF, Ceacero TM, Cyrillo JNDSG, et al. Genome-wide association study for carcass traits in an experimental Nelore cattle population. PLoS One. 2017;12:e0169860.

52. Hay EH, Roberts A. Genome-wide association study for carcass traits in a composite beef cattle breed. Livest Sci. 2018;213:35-43.

53. Purfield DC, Evans RD, Berry DP. Reaffirmation of known major genes and the identification of novel candidate genes associated with carcassrelated metrics based on whole genome sequence within a large multibreed cattle population. BMC Genomics. 2019;20:720.

54. Trychta KA, Bäck S, Henderson MJ, Harvey BK. KDEL receptors are differentially regulated to maintain the ER proteome under calcium deficiency. Cell Rep. 2018;25(1829-40):e6.

55. Brøndum RF, Su G, Janss L, Sahana G, Guldbrandtsen B, Boichard D, et al. Quantitative trait loci markers derived from whole genome sequence data increases the reliability of genomic prediction. J Dairy Sci. 2015;98:4107-16

56. Erbe M, Hayes BJ, Matukumalli LK, Goswami S, Bowman PJ, Reich CM, et al. Improving accuracy of genomic predictions within and between dairy cattle breeds with imputed high-density single nucleotide polymorphism panels. J Dairy Sci. 2012;95:4114-29.

57. Zhang X, Lourenco D, Aguilar I, Legarra A, Misztal I. Weighting strategies for single-step genomic BLUP: an iterative approach for accurate calculation of GEBV and GWAS. Front Genet. 2016;7:151. 
58. Fragomeni BO, Lourenco DA, Masuda Y, Legarra A, Misztal I. Incorporation of causative quantitative trait nucleotides in single-step GBLUP. Genet Sel Evol. 2017:49:59

59. Khansefid M, Pryce JE, Goddard ME. Increasing the accuracy of genomic prediction for residual feed intake in dairy cattle by using SNPS associated with RFI in beef cattle. Proc Assoc Advmt Anim Breed Genet. 2017;22:153-6.

60. Bolormaa S, Chamberlain AJ, Khansefid M, Stothard P, Swan AA, Mason $B$, et al. Accuracy of imputation to whole-genome sequence in sheep. Genet Sel Evol. 2019;51:1.

61. Evans LM, Tahmasbi R, Vrieze SI, Abecasis GR, Das S, Gazal S, et al. Comparison of methods that use whole genome data to estimate the heritability and genetic architecture of complex traits. Nat Genet. 2018;50:737-45.
62. Meuwissen THE, Goddard ME. Accurate prediction of genetic values for complex traits by whole genome resequencing. Genetics. 2010;185:623-31.

63. Wang J, Zhou Z, Zhang Z, Li H, Liu D, Zhang Q, et al. Expanding the BLUP alphabet for genomic prediction adaptable to the genetic architectures of complex traits. Heredity. 2018;121:648-62.

\section{Publisher's Note}

Springer Nature remains neutral with regard to jurisdictional claims in published maps and institutional affiliations.
Ready to submit your research? Choose BMC and benefit from:

- fast, convenient online submission

- thorough peer review by experienced researchers in your field

- rapid publication on acceptance

- support for research data, including large and complex data types

- gold Open Access which fosters wider collaboration and increased citations

- maximum visibility for your research: over $100 \mathrm{M}$ website views per year

At BMC, research is always in progress.

Learn more biomedcentral.com/submissions 